\title{
Convergence and optimality of a new iterative price-based scheme for distributed coordination of flexible loads in the electricity market
}

\author{
Antonio De Paola, David Angeli and Goran Strbac
}

\begin{abstract}
This paper proposes a novel distributed control strategy for large-scale deployment of flexible demand. The devices are modelled as competing players that respond to iterative broadcasts of price signals, scheduling their power consumption to operate at minimum cost. By describing their power update at each price broadcast through a multi-valued discrete-time dynamical system and by applying Lyapunov techniques, it is shown that the proposed control strategy always converges to a stable final configuration, characterized as a Wardrop (or aggregative) equilibrium. It is also proved that such equilibrium is socially efficient and optimizes some global performance index of the system (e.g. minimizes total generation costs). These results are achieved under very general assumptions on the electricity price and for any penetration level of flexible demand. Practical implementation of the proposed scheme is discussed and tested in simulation on a future scenario of the UK-grid with large numbers of flexible loads.
\end{abstract}

\section{INTRODUCTION}

Power systems are undergoing transformations of unprecedented scale and impact. One of the most significant changes is the increasing diffusion of new typologies of loads, such as electric vehicles and 'smart' appliances, that give private customers a certain flexibility in their power consumption. Such flexibility can potentially be exploited for multiple objectives, such as reducing energy costs of private households or contributing to the safety and reliability of the system [1], [2]. To fully achieve these benefits, it is necessary to devise a proper coordination of the flexible loads, balancing the (potentially conflicting) objectives of private customers and system operator and avoiding undesirable effects such as rebound peaks or synchronicity phenomena. As centralized techniques [3] may not be suitable for large-scale applications with millions of devices, distributed schemes are receiving increasing attention. A wide range of different approaches has been proposed, including Lagrange relaxation [4], stochastic pricing [5] and distributed optimization [6].

This work adopts a game-theoretical framework, which allows to explicitly capture the conflicting interactions of the new loads, preserving customers' full control over their power consumption while seeking an efficient resource allocation within the electricity market. The flexible devices are modelled as greedy agents that schedule an assigned task in order to complete it at minimum cost. The agents interact through the changes in demand (and electricity price) associated to their aggregate power consumption. In order to

A. De Paola, D. Angeli and G. Strbac are with the Department of Electrical and Electronic Engineering, Imperial College London, London, SW7 2AZ UK (e-mail: ad5709@imperial.ac.uk; d.angeli@imperial.ac.uk; g.strbac@imperial.ac.uk). This work was supported by the Leverhulme Trust under Grant [ECF-2016-394] obtain a fair and stable solution, the Wardrop equilibrium is considered as primary design objective: at the final configuration each device (assumed to have negligible market power) has no unilateral interest in changing its power scheduling. It is shown that this solution not only satisfies the individual agents but it is also socially efficient and corresponds to reduced generation costs and flattened profiles of demand.

A substantial amount of research has applied game theory to the problem of flexible demand deployment, with particular attention to distributed iterative schemes for coordination of electric vehicles. It has been shown that, with such approaches, it is possible to converge to equilibrium by introducing additional quadratic terms in the cost function of the devices [7], [8] or under some conditions on the number of loads and the electricity price [9], [10]. These works consider large populations of devices, assuming that the power consumption by the individual loads is significantly smaller than total demand and therefore has negligible impact on electricity prices. This scenario, analytically characterized as a Wardrop equilibrium, can be interpreted in a traditional Nash sense when the number of players goes to infinity. Recent studies have investigated the existence of pure Nash equilibria in similar contexts [11] and have assessed their social efficiency [12].

This paper proposes a novel design methodology for distributed coordination of flexible demand. On the basis of the initial analysis and preliminary results presented in [13], the iterative power scheduling update by the flexible appliances is modelled as the evolution of a discretetime dynamical system, described by a multi-valued correspondence. This allows a rigorous theoretical analysis that, through the application of established stability tools (e.g. Lyapunov methods), derives fundamental equilibrium results. It is shown that the proposed scheme induces a Wardrop equilibrium in the electricity market for all penetration levels of flexible demand and it does not require additional terms in the cost function of the devices nor precise knowledge of the electricity price function. The scheme is straightforward to implement in practical contexts by letting each device iteratively perform advantageous power swaps according to updated price signals. It is also demonstrated that the achieved stable solution is socially efficient and maximizes some global functional of aggregate demand, quantifying for example the total generation costs of the system.

The paper is structured as follows: Section II models the flexible loads and their interactions with the electricity market while Section III describes the chosen game-theoretical framework and Wardrop equilibrium concept. The distributed 
scheme for flexible demand coordination and its convergence and optimality properties are presented in Section IV, testing its performance in the simulations of Section V.

\section{Modelling of Flexible Demand And ELECTRICITY MARKET}

A population $\mathscr{N}=\{1, \ldots, N\}$ of price-responsive devices operate over the discrete time interval $\mathscr{T}=\{1, \ldots, T\}$. Each device $j \in \mathscr{N}$ can be described by three quantities: the amount of energy $E_{j}$ required to complete its task, its rated power $P_{j}$ and the time interval $\mathscr{A}_{j} \subseteq \mathscr{T}$ during which it is available to operate. We denote by $u_{j, t}$ the power consumption of device $j$ at time $t$ and by $u_{j}=\left[u_{j, 1}, \ldots, u_{j, T}\right] \in \mathbb{R}^{T}$ its scheduled power profile over the considered time horizon. Representing by $\mathbb{1}_{x}$ the indicator function, the set $\mathscr{U}_{j}$ of feasible power profiles for the device $j$ can be defined as:

$\mathscr{U}_{j}:=\left\{u_{j}: \sum_{t=1}^{T} u_{j, t} \Delta t=E_{j}, \quad 0 \leq u_{j, t} \leq P_{j} \cdot \mathbb{1}_{\mathscr{A}_{j}}(t) \forall t \in \mathscr{T}\right\}$.

where $\Delta t$ denotes the chosen time-discretization step. The first condition in (1) ensures that the total energy consumed by the device $j$ (equal to the sum over time of the power $u_{j, t}$ multiplied by the time step $\Delta t$ ) corresponds to the amount $E_{j}$ required for task completion. The second condition in (1) dictates that the consumed power at each time $t$ cannot be greater than $P_{j}$ and must be equal to zero when $t \notin \mathscr{A}_{j}$ and the device $j$ is not available to operate. The notation adopted so far can be extended, representing by $u=\left[u_{1}, \ldots, u_{N}\right] \in \mathbb{R}^{N T}$ the scheduled power profile of the whole population and by $\mathscr{U}=\mathscr{U}_{1} \times \mathscr{U}_{2} \times \cdots \times \mathscr{U}_{N}$ the corresponding feasibility set.

Assumption 1: The power scheduling problem is supposed to be feasible and the parameters $\left(E_{j}, P_{j}, \mathscr{A}_{j}\right)$ of each device $j$ are such that $\mathscr{U} \neq \varnothing$.

The electrical appliances are considered to be priceresponsive: they exploit their flexibility so as to consume power at the cheapest hours of the day, completing their task at minimum energy cost. For a certain price vector $p=\left[p_{1}, \ldots, p_{T}\right] \in \mathbb{R}^{T}$, the cost $C_{j}$ sustained by the device $j$ for task completion can be expressed as:

$$
C_{j}=\sum_{t=1}^{T} p_{t} \cdot u_{j, t} \cdot \Delta t
$$

Each term of the sum in (2) corresponds to the energy cost of the device $j$ at time $t$ and it is equal to the product of the electricity price $p_{t}$ by the consumed energy $u_{j, t} \cdot \Delta t$.

In the present work, the electricity market has been abstracted by a price function $\Pi$ of aggregate power demand. At a certain time instant $t \in \mathscr{T}$, the electricity price $p_{t}$ corresponds to $p_{t}=\Pi\left(D_{t}(u)\right)$. The term $D_{t}(u)$ denotes the total power demand at time $t$ when the flexible appliances population applies the scheduling $u$. It can be expressed as the sum of the total power $d_{t}$ consumed by the inflexible loads (assumed to be known a priori) and the aggregate power consumption of the price-responsive appliances:

$$
D_{t}(u)=d_{t}+\sum_{j=1}^{N} u_{j, t}
$$

Assumption 2: The price function $\Pi(D)$ is strictly monotone increasing with respect to $D$.

It is assumed that electricity is more expensive when more power needs to be generated in order to accommodate higher levels of demand. The presented analysis is still valid as long as the price function can be expressed as $p_{t}=\Pi\left(\alpha_{t}+\right.$ $\left.\sum_{j=1}^{N} u_{j, t}\right)$, where $\alpha$ is an arbitrary signal over time.

\section{GAME-TheOreticAl Formulation AND WARDROP EQUILIBRIUM AS DESIGN OBJECTIVE}

The price-responsive appliances are modelled as greedy agents that compete for power consumption at times with cheapest electricity. To devise a suitable coordination scheme for these loads, it is crucial to take into account rebound effects and synchronicity phenomena. If all devices try to operate when prices are low, they will increase the aggregate demand and the electricity prices at those times, thus making their initial strategies suboptimal. To account for the appliances interaction and properly design their coordination, the following game-theoretical framework is considered:

- Players: The $\mathscr{N}=\{1, \ldots, N\}$ price-responsive loads.

- Strategies: For each player $j \in \mathscr{N}$, the set $\mathscr{U}_{j}$ of feasible power schedules guaranteeing task completion.

- Objective function: Minimization of the actual energy cost $\bar{C}_{j}$ sustained by the individual device $j$. This quantity depends, through the price function $\Pi$, on the total power demand $D(u)$, defined in (3):

$$
\bar{C}_{j}:=\sum_{t=1}^{T} \Pi\left(D_{t}(u)\right) \cdot u_{j, t} \cdot \Delta t .
$$

The following equilibrium notion is now presented for the aforementioned game:

Definition 1: Consider a feasible vector of scheduled power profiles $u^{*} \in \mathscr{U}$. This corresponds to a Wardrop (or aggregative) equilibrium in the electricity market if the following conditions are satisfied for all $j \in \mathscr{N}$ :

$$
\sum_{t=1}^{T} \Pi\left(D_{t}\left(u^{*}\right)\right) \cdot u_{j, t}^{*} \cdot \Delta t=\min _{u_{j} \in \mathscr{U}_{j}} \sum_{t=1}^{T} \Pi\left(D_{t}\left(u^{*}\right)\right) \cdot u_{j, t} \cdot \Delta t
$$

where the aggregate demand profile $D_{t}\left(u^{*}\right)$ is equal to:

$$
D_{t}\left(u^{*}\right)=d_{t}+\sum_{j=1}^{N} u_{j, t}^{*}
$$

Remark 1: It is assumed in Definition 1 that the individual device $j$ neglects its market power and performs its cost optimization considering a fixed electricity price, equal to $\Pi\left(D\left(u^{*}\right)\right)$. This approximation is acceptable in the context of large populations of domestic appliances, whose power consumption is considerably smaller than total demand. When the number of players tends to infinity, the above equilibrium notion corresponds to a Nash equilibrium in the classical sense.

The Wardrop equilibrium is considered a primary objective for distributed coordination of flexible appliances, as it corresponds to a stable and fair solution for all the agents, which cannot reduce their final energy costs by unilaterally 
changing their operation strategy. The proposed equilibrium notion is also socially efficient: as formally proved in the next section, it optimizes some global performance index of the system.

To derive equivalent equilibrium conditions for the subsequent analysis, the following quantity is introduced:

$$
\gamma\left(u, j, t^{+}, t^{-}\right):=\left(D_{t^{-}}(u)-D_{t^{+}}(u)\right)\left(P_{j}-u_{j, t^{+}}\right) \cdot u_{j, t^{-}} .
$$

As clarified below, $\gamma$ is used to determine whether, for a certain scheduling $u$, the single device $j$ can reduce its energy cost by swapping power from $t^{-}$to $t^{+}$.

Proposition 1: A feasible vector of scheduled power profiles $u^{*} \in \mathscr{U}$ fulfils (5) and corresponds to a Wardrop equilibrium according to Definition 1 if and only if:

$$
\begin{array}{r}
\gamma\left(u^{*}, j, t^{+}, t^{-}\right) \leq 0 \quad \forall j \in \mathscr{N}, \forall\left(t^{+}, t^{-}\right) \in \mathscr{A}_{j} \times \mathscr{A}_{j} . \\
\text { Proof: Any feasible } u_{j} \in \mathscr{U}_{j} \text { can be expressed as: }
\end{array}
$$

$$
u_{j}=u_{j}^{*}+\sum_{p=1}^{P} \delta_{p}
$$

where each term $\delta_{p}: \mathscr{T} \rightarrow \mathbb{R}$, for some $t_{p}^{+}, t_{p}^{-} \in \mathscr{A}_{j}$ and $\Delta_{p}>$ 0 , has the following expression:

$$
\delta_{p, t}=\Delta_{p} \cdot \mathbb{1}_{\left\{t_{p}^{+}\right\}}(t)-\Delta_{p} \cdot \mathbb{1}_{\left\{t_{p}^{-}\right\}}(t) .
$$

This formulation is possible since all feasible power profiles in $\mathscr{U}_{j}$ (including $u_{j}$ and $u_{j}^{*}$ ) have equal total sum. It can also be assumed, without loss of generality, that each $\delta_{p}$ corresponds to a feasible power swap between the time instants $t_{p}^{+}$and $t_{p}^{-}$and therefore we have:

$$
0<\Delta_{p} \leq \min \left(P_{j}-u_{j, t_{p}^{+}}^{*}, u_{j, t_{p}^{-}}^{*}\right) .
$$

The cost function in the right-hand side of (5) can then be rewritten as:

$$
\begin{array}{r}
\sum_{t=1}^{T} \Pi\left(D_{t}\left(u^{*}\right)\right) \cdot u_{j, t} \cdot \Delta t=\sum_{t=1}^{T} \Pi\left(D_{t}\left(u^{*}\right)\right) \cdot u_{j, t}^{*} \cdot \Delta t \\
+\sum_{p=1}^{P}\left[\Pi\left(D_{t_{p}^{+}}\left(u^{*}\right)\right)-\Pi\left(D_{t_{p}^{-}}\left(u^{*}\right)\right)\right] \cdot \Delta_{p} \Delta t .
\end{array}
$$

Note that, if $u_{j}^{*}$ does not fulfill (5), from (11) and (12) there must exist $t_{p}^{+}=t^{+}$and $t_{p}^{-}=t^{-}$with the following properties:

$$
\Pi\left(D_{t^{+}}\left(u^{*}\right)\right)<\Pi\left(D_{t^{-}}\left(u^{*}\right)\right) \quad u_{j, t^{+}}^{*}<P_{j} \quad u_{j, t^{-}}^{*}>0 .
$$

Given the monotonicity of the price function $\Pi$ established in Assumption 2, from (13) it follows:

$$
\gamma\left(u^{*}, j, t^{+}, t^{-}\right)=\left(D_{t^{-}}\left(u^{*}\right)-D_{t^{+}}\left(u^{*}\right)\right) \cdot\left(P_{j}-u_{j, t^{+}}^{*}\right) \cdot u_{j, t^{-}}^{*}>0
$$

which contradicts (8) and concludes the proof.

\section{Distributed CoORdinAtion StRategy}

The appliances are coordinated through iterative updates of their power scheduling. A distributed paradigm is adopted: a central entity sequentially broadcasts a price signal to each device, which in turn performs an advantageous power swap to reduce its energy cost.

\section{A. Power Update as Evolution of Dynamical System}

The iterative changes in the scheduled power profiles of the flexible appliances are first described through a discretetime dynamical system:

$$
u(0)=u^{0} \quad u(k+1) \in F(u(k))
$$

where $F: \mathscr{U} \mapsto \mathscr{U}$ is a multi-valued correspondence and $u(k) \in \mathscr{U}$ represents the power consumption scheduled by the whole population of flexible loads after $k$ iterations of the proposed coordination strategy. We argue that this formulation, formally more rigorous than the pseudo-algorithm representation usually adopted in similar works on the subject, allows to directly apply a wide range of theoretical tools (e.g. Lyapunov stability theory) and derive fundamental results in the context of flexible demand integration. Practical implementation methods are discussed in Section IV-B.

In order to formally characterize $F$, the following quantities are preliminarily introduced:

$$
S_{j}(u):=\underset{\left(t^{+}, t^{-}\right) \in \mathscr{A}_{j} \times \mathscr{A}_{j}}{\arg \max } \gamma\left(u, j, t^{+}, t^{-}\right)
$$

$\Delta\left(u, j, t^{+}, t^{-}\right):=\min \left(P_{j}-u_{j, t^{+}}, u_{j, t^{-}}, \frac{\left\lfloor D_{t^{-}}(u)-D_{t^{+}}(u)\right\rfloor_{+}}{2}\right)$

where $\lfloor x\rfloor_{+}$denotes positive part of $x$. The set $S_{j}(u)$ associates to a device $j \in \mathscr{N}$ the pairs of time instants $s_{j}=$ $\left(t_{j}^{+}, t_{j}^{-}\right)$in its availability interval that maximize the function $\gamma$ under the current $u$ and can be considered for cost-reducing power shifts. The maximum power that can be swapped between these two times is quantified by $\Delta$ in (16).

A multi-valued correspondence $F_{j}: \mathscr{U} \mapsto \mathscr{U}$ is first defined for each device $j \in \mathscr{N}$ :

$$
F_{j}(u)=\bigcup_{s_{j} \in S_{j}(u)} f^{\left(s_{j}\right)}(u)=\bigcup_{s_{j} \in S_{j}(u)}\left[f_{1,1}^{\left(s_{j}\right)}(u), \ldots, f_{N, T}^{\left(s_{j}\right)}(u)\right]
$$

where the single component $f_{i, t}^{\left(s_{j}\right)}(u)$ of $f^{\left(s_{j}\right)}(u)$ in (17) has the following expression when $s_{j}=\left(t_{j}^{+}, t_{j}^{-}\right)$:

$f_{i, t}^{\left(s_{j}\right)}(u)=u_{i, t}+\Delta\left(u, j, t_{j}^{+}, t_{j}^{-}\right) \cdot \mathbb{1}_{\{j\}}(i)\left[\mathbb{1}_{\left\{t_{j}^{+}\right\}}(t)-\mathbb{1}_{\left\{t_{j}^{-}\right\}}(t)\right]$.

When $F_{j}$ is applied, a single element $s_{j}=\left(t_{j}^{+}, t_{j}^{-}\right) \in S_{j}(u)$ is selected, and the $j$-th device shifts power from the time instant $t_{j}^{-}$to $t_{j}^{+}$, as detailed in (18). The amount of shifted power is determined by $\Delta$ in (16), which guarantees that the new power profile is feasible and that the power swap occurs only if demand (and therefore price) at $t_{j}^{-}$is higher then at $t_{j}^{+}$. The other devices $i \in \mathscr{N} \backslash\{j\}$ preserve their previous power scheduling. The complete multi-valued correspondence $F$ in (14) corresponds to an iterative power update over the whole population of appliances and can be represented as the composition of $N$ mappings $F_{j}$, one for each device $j \in \mathscr{N}$ :

$$
F(u):=\left(F_{N} \circ \cdots \circ F_{1}\right)(u) .
$$

Definition 2: Given the dynamical system (14) with $F$ as defined in (19), its solution set $\Phi$ is the following:

$$
\Phi:=\left\{\phi: \mathbb{N}^{+} \rightarrow \mathscr{U}: \phi(k+1) \in F(\phi(k)) \forall k \in \mathbb{N}^{+}\right\} .
$$


The properties of system (14) and its solutions are studied with Lyapunov techniques, demonstrating that the proposed update strategy always converges to the set of Wardrop equilibria. To this end, the following preliminary results are provided:

Proposition 2: Consider a functional $V: \mathscr{U} \rightarrow \mathbb{R}^{+}$of the following type:

$$
V(u):=\sum_{t=1}^{T} g\left(D_{t}(u)\right)
$$

where $g$ is a positive strictly-convex function.

1) For all solutions $\phi \in \Phi$ of system (14), as defined in (20), there exists $V_{\infty} \in \mathbb{R}^{+}$such that:

$$
\lim _{k \rightarrow \infty} V(\phi(k))=V_{\infty}
$$

2) If $V(\phi(k+1))=V(\phi(k))$, then $\phi(k+1)=\phi(k)$.

Proof: See Appendix I.

The main results of this section can now be presented:

Theorem 1: Denote by $\Omega^{*} \subseteq \mathscr{U}$ the set of Wardrop equilibria as expressed in Definition 1 and by $|x|_{\Gamma}$ the distance between a point $x \in \mathscr{U}$ and a set $\Gamma \subseteq \mathscr{U}$. For each solution $\phi \in \Phi$ of (14) as defined in (20), it holds:

\section{Proof: See Appendix II.}

$$
\lim _{k \rightarrow+\infty}|\phi(k)|_{\Omega^{*}}=0
$$

From Theorem 1 we can conclude that all solutions of (14) asymptotically converge to the set of Wardrop equilibria in the electricity market. We wish to emphasize that this crucial result is achieved for all penetration levels of flexible demand and all parameters of the devices, as long as Assumption 1 is verified and the scheduling problem is feasible. Note also that specific knowledge of the price function $\Pi$ is not required and only its monotonicity with respect to power demand needs to be assumed. Before discussing in Section IV-B how to achieve these results in practical implementations, a global property of the equilibrium is presented.

Theorem 2: Assume a positive strictly-convex $g$ for the functional $V$ in (21). For any $u^{*} \in \Omega^{*}$ corresponding to a Wardrop equilibrium and any feasible $u \in \mathscr{U}$, it holds:

\section{Proof: See Appendix III.}

$$
V\left(u^{*}\right) \leq V(u)
$$

It follows from Theorem 2 that the equilibrium notion presented in Definition 1 not only corresponds to a stable solution for the greedy optimization of the individual flexible agents but it is also socially efficient, i.e. optimizes some global performance index of the system. For example, the functional $V$ can be used to quantify the total generation costs in the grid or the achieved flattening of aggregate demand. This is an important result in the context of future largescale deployment of flexible demand in power systems. In particular, it shows that integration of large populations of price-responsive devices can be performed in a distributed price-based setting while preserving the efficiency and the reliability of the grid, with limited centralized intervention.

\section{B. Practical Implementation}

The power scheduling update described by $F$ in Section IV-A can be implemented in practice through a bi-directional communication scheme between the system operator and the flexible loads. The general idea is that, on the basis of a price signal broadcast by a central entity, each device $j$ can choose a power swap that reduces its energy cost and corresponds to the application of a certain $f^{\left(s_{j}\right)}(u) \in F_{j}(u)$. Three distinct phases can be considered for the implementation:

1) Initialization phase: The initial power scheduling $u(0)$ is obtained by broadcasting a price signal $p(0): \mathscr{T} \rightarrow \mathbb{R}^{+}$ to all agents and letting each device $j$ schedule its power consumption $u_{j}(0)$ so as to minimize its expected cost:

$$
u_{j}(0) \in \underset{u_{j} \in \mathscr{U}_{j}}{\arg \min } \sum_{t=1}^{T} p_{t}(0) \cdot u_{j, t} \cdot \Delta t .
$$

The scheduling $u(0)$ is communicated to the central entity, which calculates the corresponding $D(u(0))$ through (3).

2) Power scheduling update (summarized in Fig. 1): The individual power updates $F_{j}$ in (19) are applied sequentially, through the following steps:

a) At the $l$-th iteration, the price signal $p(l)=\Pi(D(u(l)))$ is broadcast to the device $j$ (Phase j.A in Fig. 1).

b) The device $j$ reduces its cost by swapping an amount of power $\Delta$ from $t_{j}^{-}$to $t_{j}^{+}$(with lower price), according to (18) with $i=j$. Since $\Pi$ is monotone, $s_{j}=\left(t_{j}^{+}, t_{j}^{-}\right)$and $\Delta$ can be calculated with minimum modifications, considering $p(l)=\Pi(D(u(l)))$ instead of $D(u(l))$. Convergence and optimality properties of the algorithm are preserved.

c) Device $j$ communicates $t_{j}^{+}, t_{j}^{-}$and $\Delta$ to the system operator (Phase j.B in Fig. 1) which in turn derives a new power demand $D(u(l+1))$.

d) Steps a)-c) are repeated for $l=l+1$ and $j=j+1$ (resetting $j$ to 1 if the final value $N$ has been reached).

3) Final results: When the devices do not update further their power scheduling (equilibrium is reached), the algorithm is stopped. The power scheduling at equilibrium is $u^{*}=u(l)$, where $l$ is the index of the last power update.

Remark 2: The presented implementation assumes that convergence (which is proved asymptotically) is actually achieved in a finite number of steps. Numerical simulations presented in Section V seem to confirm this hypothesis. Nevertheless, a stopping criterion can be added in order to terminate the algorithm when the potential cost reduction achievable by the devices is negligible.

We are aware that the proposed implementation could be particularly time-consuming when large number of devices are considered, as a bidirectional communication channel needs to be established (potentially more than one time) with each device. Alternative one-shot schemes have already been derived, allowing the system operator to internally perform most of the calculations and coordinate the flexible appliances by broadcasting a single price signal (different in general for each load). These techniques, discussed in detail in [13], ensure significantly reduced execution times 


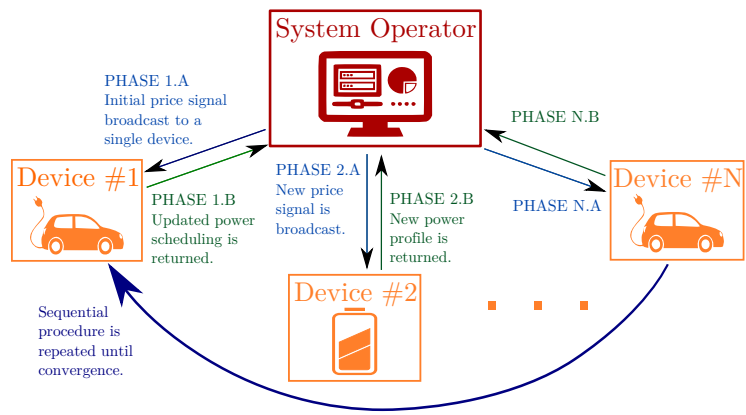

Fig. 1. Bi-directional scheme for practical implementation of power scheduling updates.

in practical contexts, at the price of a negligible equilibrium approximation at the final solution.

\section{Simulation Results}

The proposed scheme for flexible demand coordination has been tested in simulation, considering a future scenario of the UK power grid. In addition to the inflexible demand $d$, chosen as the total power consumption of a typical winter day [14], the charging of $N=2 \cdot 10^{6}$ electric vehicles (EVs) is performed. We assume that the EVs have equal power rating $P_{j}=P=10 \mathrm{KW}$ while their parameters $E_{j}$ are generally different and depend on the state of charge of their batteries when they are plugged into the grid. The chosen values of $E_{j}$ are normally distributed, with mean $\mu_{E}=30 \mathrm{KWh}$ and standard deviation $\sigma_{E}=1.5 \mathrm{KWh}$. To determine the EVs' availability windows $\mathscr{A}_{j}$, it is assumed that the $j$-th EV is plugged in at time $t_{j}$ and remains connected for $c_{j}$ hours where $t_{j}$ and $c_{j}$ are also normally distributed, with the following mean $\mu$ and standard deviation $\sigma$ :

$$
\mu_{t}=21: 30 \mathrm{~h} \quad \sigma_{t}=1.5 \mathrm{~h} \quad \mu_{c}=10 \mathrm{~h} \quad \sigma_{c}=1 \mathrm{~h} .
$$

The procedure described in Section IV-B has been simulated assuming that each device $j$, having received a price signal from the system operator, can perform multiple power swaps in order to reduce its energy cost. The calculations have required about 13 minutes to be completed on a standard laptop machine. The resulting demand profiles $D(u(l))$ after $l$ power updates by the individual devices are represented in Fig. 2. As previously mentioned, when appliances respond to the broadcast price of inflexible demand $d$ (bluedashed line), the resulting aggregate power consumption $D(u(0))$ of the system (black-dashed line) has a substantial peak, with most of the vehicles charging at high electricity prices. Through iterative power swaps, the demand profile is gradually flattened. At $l=20 \cdot 10^{5}=N$, after all EVs have received an updated price signal and modified their power scheduling once, no peak appears in the resulting demand profile (magenta line). The final power scheduling (corresponding to a Wardrop equilibrium) is obtained at $l=$ $40 \cdot 10^{5}=2 \mathrm{~N}$, when all EVs have rescheduled their charging twice. Note that the corresponding demand profile (red dotdashed line) is mostly flat, with a small valley between $8: 00 \mathrm{~h}$

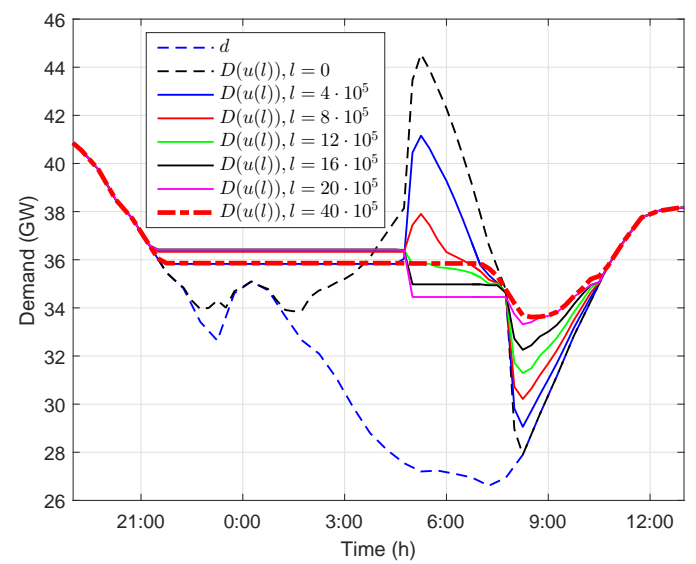

Fig. 2. Profiles of aggregate power demand after $l$ power updates of the proposed coordination strategy.

and 10:00h which is not filled as most of the vehicles are not plugged in at such times and cannot consume power.

The performance of the proposed control strategy has been compared to alternative cases with no appliances coordination. Specifically, a 'time-greedy' (TG) scenario has been considered, assuming that each device aims at completing its task as soon as possible. The scenario 'price-greedy' (PG) has also been analysed, with each appliance scheduling its power consumption on the basis of a single price signal, equal to the electricity price of inflexible demand $d$. The resulting power demand profiles (respectively $D^{T G}$ and $D^{P G}$ ) are compared in Fig. 3 with $D\left(u^{*}\right)$, obtained with the proposed coordination scheme. Note that, in the absence of distributed coordination between the loads, their operation strategies introduce new demand peaks in the system and do not correspond to a Wardrop equilibrium, as most of the devices could shift their power consumption to the new valleys with cheaper electricity appearing in $D^{T G}$ and $D^{P G}$. It has been calculated that, with the induced power scheduling $u^{*}$, the average energy cost for the single device is $27 \%$ and $13 \%$ smaller than in the TG and PG case. We also wish to emphasize that $u^{*}$ ensures lower generation costs for the system. As discussed in Section IV-A, this quantity is actually minimized by $u^{*}$ under very general assumptions.

\section{CONCLUSIONS}

This paper presents a novel distributed technique for largescale deployment of flexible demand in power systems. By modelling the power scheduling update of the priceresponsive appliances as the evolution of a discrete-time dynamical system, it is possible to use Lyapunov techniques and demonstrate that the proposed coordination scheme converges to a Wardrop equilibrium for all penetration levels of flexible demand. It is also proved that the final solution is socially efficient and optimizes some global index of the system, such as total generation costs. Finally, the effectiveness of the proposed control strategy has been demonstrated in simulations, considering future scenarios of the UK power grid with large populations of flexible loads. 


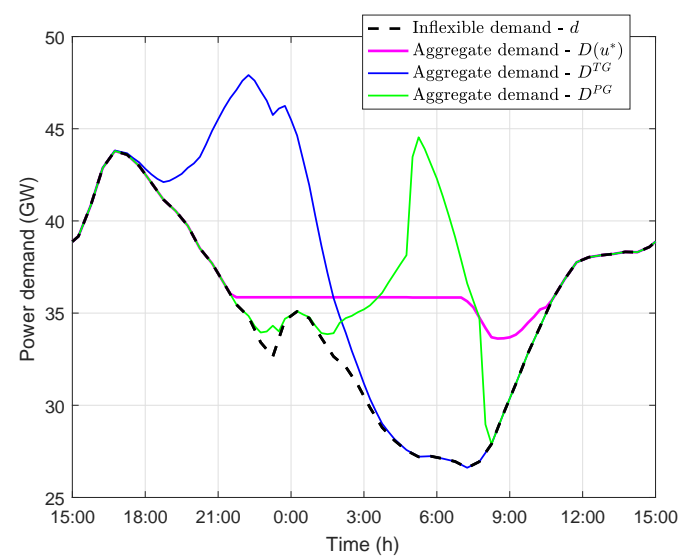

Fig. 3. Aggregate demand profiles for different power consumption strategies of the flexible loads.

\section{APPENDIX I}

ProOF OF PROPOSITION 2

For the first statement, since $V$ is bounded below by zero, it suffices to show that $V(\phi(k+1)) \leq V(\phi(k))$ or, equivalently:

$$
V(y) \leq V(u) \quad \forall y \in F(u), \forall u \in \mathscr{U} .
$$

As $F$ in (19) is the composition of $N$ correspondences $F_{j}$, this is verified if:

$$
V(y) \leq V(u) \quad \forall y \in F_{j}(u), \forall u \in \mathscr{U}, \forall j \in \mathscr{N} .
$$

To prove (27), it can be shown that the following holds for all $s_{j}=\left(t_{j}^{+}, t_{j}^{-}\right) \in S_{j}(u)$ :

$$
\begin{array}{ll}
V(y)=V\left(f^{\left(s_{j}\right)}(u)\right)<V(u) & \text { if } \gamma\left(u, j, t_{j}^{+}, t_{j}^{-}\right)>0 \\
V(y)=V\left(f^{\left(s_{j}\right)}(u)\right)=V(u) & \text { if } \gamma\left(u, j, t_{j}^{+}, t_{j}^{-}\right) \leq 0
\end{array}
$$

When $\gamma\left(u, j, t_{j}^{+}, t_{j}^{-}\right)>0$, since $u_{j, t_{j}^{+}} \leq P_{j}$ and $u_{j, t_{j}^{-}} \geq 0$ by definition, as a result of (7) it holds:

$$
D_{t_{j}^{-}}(u)>D_{t_{j}^{+}}(u) \quad u_{j, t_{j}^{+}}<P_{j} \quad u_{j, t_{j}^{-}}>0 .
$$

From (16) we have that $\Delta\left(u, j, t_{j}^{+}, t_{j}^{-}\right)$, hereby denoted simply as $\Delta$, is also positive. In the present case, given expression (18) for the components of $y=f^{\left(s_{j}\right)}(u) \in F_{j}(u)$, it holds:

$$
\begin{aligned}
D_{t_{j}^{+}}(u)<D_{t_{j}^{+}}(u)+\Delta & =D_{t_{j}^{+}}(y) \leq D_{t_{j}^{-}}(y)=D_{t_{j}^{-}}(u)-\Delta<D_{t_{j}^{-}}(u) . \\
D_{t}(u) & =D_{t}(y) \quad \forall t \in \mathscr{T} \backslash\left\{t_{j}^{+}, t_{j}^{-}\right\} .
\end{aligned}
$$

The values of $V$ at $u$ and $y=f^{\left(s_{j}\right)}(u)$ are now compared:

$$
\begin{aligned}
V(y)-V(u) & =g\left(D_{t_{j}^{+}}(y)\right)-g\left(D_{t_{j}^{+}}(u)\right)+g\left(D_{t_{j}^{-}}(y)\right)-g\left(D_{t_{j}^{-}}(u)\right) \\
& =\int_{0}^{\Delta} g^{\prime}\left(D_{t_{j}^{+}}(u)+x\right)-g^{\prime}\left(D_{t_{j}^{-}}(u)-x\right) d x<0
\end{aligned}
$$

where the inequality follows from (29a) and the strict convexity of $g$. Having verified (28a), condition (28b) is now considered. With similar arguments, it can be shown from (7) that $\Delta\left(u, j, t_{j}^{+}, t_{j}^{-}\right)$as defined in (16) is equal to zero when $\gamma\left(u, j, t_{j}^{+}, t_{j}^{-}\right) \leq 0$. It follows from (18) that $y=$ $f^{\left(s_{j}\right)}(u)=u$, implying that $V(y)=V(u)$ and thus proving (28b) and the first proposition statement. To verify the second claim consider that, since $V$ is nondecreasing when any $F_{j}$ is applied, (28b) must hold for all $j$ when $u=\phi(k)$ and $V(\phi(k+1))=V(\phi(k))$. As previously proved, this implies $\Delta\left(u, j, t_{j}^{+}, t_{j}^{-}\right)=0$ for all $j$ and therefore $\phi(k+1)=\phi(k)$.

\section{APPENDIX II}

\section{PROOF OF THEOREM 1}

Consider the $\omega$-limit set $\Omega(\phi)$ associated to the solution $\phi$ and defined as:

$$
\Omega(\phi):=\left\{u_{\infty}: \exists\left\{k_{n}\right\}_{n \in \mathbb{N}}, \lim _{n \rightarrow \infty} k_{n}=\infty, \lim _{n \rightarrow \infty} \phi\left(k_{n}\right)=u_{\infty}\right\} .
$$

To prove the theorem, given the definition of distance to a set, it is sufficient to show the following:

$$
\begin{gathered}
\lim _{k \rightarrow \infty}|\phi(k)|_{\Omega(\phi)}=0 \\
\Omega(\phi) \subseteq \Omega^{*} .
\end{gathered}
$$

A preliminary result is initially demonstrated.

Graph of $F$ is closed: It is shown that $F$ in (19) has closed graph. The same property is first proven for the graph $G_{j}$ of $F_{j}$ in (17), with the following expression:

$$
\begin{aligned}
G_{j} & =\left\{\left(u, f^{\left(s_{j}\right)}(u)\right): u \in \mathscr{U}, f^{\left(s_{j}\right)}(u) \in F_{j}(u)\right\} \\
& =\bigcup_{s_{j} \in \mathscr{A}_{j} \times \mathscr{A}_{j}} G^{\left(s_{j}\right)}=\bigcup_{s_{j} \in \mathscr{A}_{j} \times \mathscr{A}_{j}}\left\{\left(u, f^{\left(s_{j}\right)}(u)\right): u \in U^{\left(s_{j}\right)}\right\} .
\end{aligned}
$$

where $U^{\left(s_{j}\right)} \subseteq \mathscr{U}$ is defined as follows:

$$
\begin{aligned}
U^{\left(s_{j}\right)}= & \left\{u \in \mathscr{U}: s_{j}=\left(t_{j}^{+}, t_{j}^{-}\right) \in S_{j}(u)\right\} \\
= & \left\{u \in \mathscr{U}: s_{j}=\left(t_{j}^{+}, t_{j}^{-}\right),\right. \\
& \left.\gamma\left(u, j, t_{j}^{+}, t_{j}^{-}\right) \geq \gamma\left(u, j, t^{+}, t^{-}\right) \forall\left(t^{+}, t^{-}\right) \in \mathscr{A}_{j} \times \mathscr{A}_{j}\right\} .
\end{aligned}
$$

The graph $G^{\left(s_{j}\right)}$ is closed since the state-space subset $U^{\left(s_{j}\right)}$ is closed (defined by a set of non-strict inequalities) and it can be verified from (18) that each component $f_{i, t}^{\left(s_{j}\right)}(u)$ of $f^{\left(s_{j}\right)}(u)$ in (17) is continuous with respect to $u$. The graph $G_{j}$ is also closed since it is the union of a finite number of closed sets. One can conclude that also $F$ has closed graph, since it is the composition of correspondences $F_{j}$ with the same property and $\mathscr{U}$ is compact [15].

Proof of convergence: Condition (32a) straightly follows from the outer semicontinuity of $F$ [16, Chapter 6.3.3], guaranteed by its graph $G$ being closed [17, Chapter 3B].

Proof of equilibrium: It is now shown that (32b) holds and all the elements in $\Omega(\phi)$ are Wardrop equilibria. From Proposition 2, for any $u_{\infty} \in \Omega(\phi)$, it holds:

$V\left(u_{\infty}\right)=V\left(\lim _{n \rightarrow \infty} \phi\left(k_{n}\right)\right)=\lim _{n \rightarrow \infty} V\left(\phi\left(k_{n}\right)\right)=\lim _{k \rightarrow \infty} V(\phi(k))=V_{\infty}$.

Given the weak-invariance of $\Omega(\phi)$, which follows from the outer semicontinuity of $F$ [16, Chapter 6.3.3], we have:

$$
F\left(u_{\infty}\right) \cap \Omega(\phi) \neq \varnothing \quad \forall u_{\infty} \in \Omega(\phi) .
$$


Therefore, from (35), there always exists $y \in F\left(u_{\infty}\right) \cap \Omega(\phi)$ such that $V(y)=V\left(u_{\infty}\right)=V_{\infty}$, with $y=u_{\infty}$ from the second part of Proposition 2. From (28b), there must exist $\bar{s}_{1}=$ $\left(\bar{t}_{1}^{+}, \bar{t}_{1}^{-}\right) \in S_{1}\left(u_{\infty}\right)$ such that:

$$
V\left(f^{\left(\bar{s}_{1}\right)}\left(u_{\infty}\right)\right)=V\left(u_{\infty}\right) \quad \gamma\left(u_{\infty}, 1, \bar{t}_{1}^{+}, \bar{t}_{1}^{-}\right) \leq 0 .
$$

If this were not the case, since $V$ is nonincreasing for all $F_{j}$, we would have $V\left(f\left(u_{\infty}\right)\right)<V\left(u_{\infty}\right)$ for all $f\left(u_{\infty}\right) \in F\left(u_{\infty}\right)$ and therefore $u_{\infty} \notin F\left(u_{\infty}\right)$, contradicting an established result. As $\gamma$ is maximized by all $s_{1} \in S_{1}\left(u_{\infty}\right)$ from (15), we have:

$$
\begin{array}{ll}
\gamma\left(u_{\infty}, 1, t_{1}^{+}, t_{1}^{-}\right) \leq 0 & \forall s_{1}=\left(t_{1}^{+}, t_{1}^{-}\right) \in S_{1}\left(u_{\infty}\right) . \\
\Delta\left(u_{\infty}, 1, t_{1}^{+}, t_{1}^{-}\right)=0 & \forall s_{1}=\left(t_{1}^{+}, t_{1}^{-}\right) \in S_{1}\left(u_{\infty}\right)
\end{array}
$$

where (37b) also implies $F_{1}\left(u_{\infty}\right)=\left\{u_{\infty}\right\}$. Recursive application of the same arguments for $j=2, \ldots, N$ yields:

$$
\gamma\left(u_{\infty}, j, t_{j}^{+}, t_{j}^{-}\right) \leq 0 \quad \forall s_{j}=\left(t_{j}^{+}, t_{j}^{-}\right) \in S_{j}\left(u_{\infty}\right), \forall j \in \mathscr{N} .
$$

To verify that $u_{\infty} \in \Omega(\phi)$ is a Wardrop equilibrium note that, given expression (15) for $S_{j}$, (38) are equivalent to (8) for $u^{*}=u_{\infty}$. This means that (32b) holds, concluding the proof.

\section{APPENDIX III \\ PROOF OF THEOREM 2}

The theorem is verified in a reordered time vector. Properties on partial sums of the demand profiles are first derived, exploiting the convexity of $g$ to prove the final result.

Time-reordering: Consider a bijective function $h$ : $\mathscr{T} \rightarrow \mathscr{T}$ and the corresponding reordered time vector $[h(1), h(2), \ldots, h(T)]$. A bar accent is used to denote any quantity evaluated over the new time variable:

$$
\begin{aligned}
\bar{u}_{j, t} & =u_{j, h(t)} & \forall j \in \mathscr{N}, \forall t \in \mathscr{T} \\
\bar{D}_{t}(u) & =D_{h(t)}(u) & \forall t \in \mathscr{T} .
\end{aligned}
$$

The functional $V$ is invariant with respect to this timereordering operation. In fact, since $h$ is bijective, we have:

$$
V(u)=\sum_{t=1}^{T} g\left(D_{t}(u)\right)=\sum_{t=1}^{T} g\left(D_{h(t)}(u)\right)=\sum_{t=1}^{T} g\left(\bar{D}_{t}(u)\right) .
$$

Moreover, for any $u$ and $u^{*}$ considered in the theorem statement, $h$ can always be chosen so as to fulfil the following:

$$
\begin{gathered}
\bar{D}_{1}\left(u^{*}\right) \leq \bar{D}_{2}\left(u^{*}\right) \leq \cdots \leq \bar{D}_{T}\left(u^{*}\right) \\
\bar{D}_{t_{1}}(u) \leq \bar{D}_{t_{2}}(u) \quad \forall\left(t_{1}, t_{2}\right): t_{1}<t_{2}, \bar{D}_{t_{1}}\left(u^{*}\right)=\bar{D}_{t_{2}}\left(u^{*}\right)
\end{gathered}
$$

Sum properties of demand profiles: Let $\bar{S}\left(u^{*}\right)$ and $\bar{S}(u)$ denote the partial sums of the demand profiles associated to $u^{*}$ and $u$ respectively, over the reordered time variable:

$$
\bar{S}_{t}\left(u^{*}\right)=\sum_{l=1}^{t} \bar{D}_{l}\left(u^{*}\right) \quad \bar{S}_{t}(u)=\sum_{l=1}^{t} \bar{D}_{l}(u) .
$$

Given $u^{*}, u \in \mathscr{U}$ with $u^{*}$ fulfilling (5), we claim:

$$
\bar{S}_{t}(u) \leq \bar{S}_{t}\left(u^{*}\right) \quad \forall t \in \mathscr{T} .
$$

A weaker property is initially demonstrated:

$$
\bar{S}_{t}(u) \leq \bar{S}_{t}\left(u^{*}\right) \quad \forall t<T: \bar{D}_{t}\left(u^{*}\right)<\bar{D}_{t+1}\left(u^{*}\right) .
$$

Since $u^{*}$ corresponds to a Wardrop equilibrium and fulfils (5), for any of the subprofiles $u_{j}^{*} \in \mathscr{U}_{j}$ we have:

$$
\begin{gathered}
\sum_{l=1}^{t} \bar{u}_{j, l}^{*} \cdot \Delta t=\min \left(E_{j}, \sum_{l \in\{1, \ldots, t\} \cap \bar{A}_{j}} P_{j} \cdot \Delta t\right) \\
\forall j \in \mathscr{N} \quad \forall t: \bar{D}_{t}\left(u^{*}\right)<\bar{D}_{t+1}\left(u^{*}\right) .
\end{gathered}
$$

where $\overline{\mathscr{A}}_{j}=\left\{t: h^{-1}(t) \in \mathscr{A}_{j}\right\}$ corresponds to the availability instants of the $j$-th device in the reordered time coordinates. To check that (45) holds, note that its left-hand side is always smaller or equal than its right-hand side. Assume by contradiction that $\sum_{l=1}^{t} \bar{u}_{j, l}^{*} \cdot \Delta t<\min \left(E_{j}, \sum_{l \in\{1, \ldots, t\} \cap \mathscr{A}_{j}} P_{j} \cdot \Delta t\right)$. This implies existence of $t^{+} \leq t$ and $t^{-}>t$ in $\overline{\mathscr{A}}_{j}$ such that:

$$
\bar{u}_{j, t^{+}}^{*}<P_{j} \quad \bar{u}_{j, t^{-}}^{*}>0 .
$$

One can then define an alternative power profile $u^{\diamond} \in \mathscr{U}_{j}$, with the following expression in the reordered coordinates:

$$
\bar{u}_{t}^{\diamond}=\bar{u}_{j, t}^{*}+\Delta u \cdot \mathbb{1}_{\left\{t^{+}\right\}}(t)-\Delta u \cdot \mathbb{1}_{\left\{t^{-}\right\}}(t)
$$

where $\Delta u=\min \left(P_{j}-\bar{u}_{j, t^{+}}^{*}, \bar{u}_{j_{2} t^{-}}^{*}\right)>0$. Since $t^{+} \leq t<t^{-}$, the inequality $\Pi\left(\bar{D}_{t^{+}}\left(u^{*}\right)\right)<\Pi\left(\bar{D}_{t^{-}}\left(u^{*}\right)\right)$ holds by construction (as we are also assuming $\left.\bar{D}_{t}\left(u^{*}\right)<\bar{D}_{t+1}\left(u^{*}\right)\right)$ and we have:

$$
\begin{aligned}
& \sum_{t=1}^{T} \Pi\left(\bar{D}_{t}\left(u^{*}\right)\right) \bar{u}_{t}^{\diamond}=\sum_{t=1}^{T} \Pi\left(\bar{D}_{t}\left(u^{*}\right)\right) \bar{u}_{j, t}^{*} \\
& \quad+\Delta u\left[\Pi\left(\bar{D}_{t^{+}}\left(u^{*}\right)\right)-\Pi\left(\bar{D}_{t^{-}}\left(u^{*}\right)\right)\right]<\sum_{t=1}^{T} \Pi\left(\bar{D}_{t}\left(u^{*}\right)\right) \bar{u}_{j, t}^{*} .
\end{aligned}
$$

This contradicts the hypothesis of $u^{*}$ fulfilling (5) as the considered quantities are invariant with respect to timereordering, confirming that (45) is satisfied. As its right-hand side represents the maximum energy consumed with any feasible $u_{j} \in \mathscr{U}_{j}$ over the reordered time interval $\{1, \ldots, t\}$, it holds:

$$
\sum_{l=1}^{t} \bar{u}_{j, l} \leq \sum_{l=1}^{t} \bar{u}_{j, l}^{*} \quad \forall j \in \mathscr{N}, \quad \forall t<T: \bar{D}_{t}\left(u^{*}\right)<\bar{D}_{t+1}\left(u^{*}\right) .
$$

Taking the sum of (47) over $j \in \mathscr{N}$ and adding the values of inflexible demand on both sides yields (44). The more general condition (43) is now proved by contradiction. Consider an interval $\mathscr{T}_{=}=\left\{t_{S}, \ldots, t_{F}\right\}$ of constant demand $\bar{D}\left(u^{*}\right)$ :

$$
\begin{gathered}
\bar{D}_{t_{1}}\left(u^{*}\right)=\bar{D}_{t_{2}}\left(u^{*}\right) \quad \forall t_{1}, t_{2} \in \mathscr{T}= \\
\bar{D}_{t_{S}-1}\left(u^{*}\right)<\bar{D}_{t_{S}}\left(u^{*}\right) \\
\bar{D}_{t_{F}}\left(u^{*}\right)<\bar{D}_{t_{F}+1}\left(u^{*}\right) .
\end{gathered}
$$

It is assumed that there exists $t_{E} \in \mathscr{T}_{=} \backslash\left\{t_{F}\right\}$ violating (43):

$$
\bar{S}_{t_{E}}(u)>\bar{S}_{t_{E}}\left(u^{*}\right) \text {. }
$$

Since $\bar{S}_{t_{S}-1}(u)<\bar{S}_{t_{S}-1}\left(u^{*}\right)$ as a result of (44) and (48b), it follows that there exists $t^{\circ} \in\left\{t_{S}, \ldots, t_{E}\right\}$ such that:

$$
\bar{D}_{t^{\circ}}(u)>\bar{D}_{t^{\circ}}\left(u^{*}\right) \text {. }
$$

Moreover, given property (41b) for the chosen timereordering, it also holds:

$$
\bar{D}_{t}(u)>\bar{D}_{t}\left(u^{*}\right) \quad \forall t \in\left\{t^{\circ}, \ldots, t_{F}\right\} \supseteq\left\{t_{E}, \ldots, t_{F}\right\} .
$$


It follows:

$\bar{S}_{t_{F}}(u)-\bar{S}_{t_{F}}\left(u^{*}\right)=\bar{S}_{t_{E}}(u)-\bar{S}_{t_{E}}\left(u^{*}\right)+\sum_{t=t_{E}+1}^{t_{F}} \bar{D}_{t}(u)-\bar{D}_{t}\left(u^{*}\right)>0$

where the inequality holds as a result of (49) and (51). Note that (52) contradicts the verified condition (44), implying that (43) also holds.

Equilibrium optimality: To prove the initial theorem statement, a more general condition is demonstrated. Consider two demand profiles $\bar{D}$ and $\bar{D}^{*}=\bar{D}\left(u^{*}\right)$ such that:

$$
\begin{gathered}
\bar{S}_{t}=\sum_{l=1}^{t} \bar{D}_{l} \leq \sum_{l=1}^{t} \bar{D}_{l}^{*}=\bar{S}_{t}^{*} \quad \forall t \in \mathscr{T} \\
\bar{S}_{T}=\sum_{l=1}^{T} \bar{D}_{l}=\sum_{l=1}^{T} \bar{D}_{l}^{*}=\bar{S}_{T}^{*}
\end{gathered}
$$

Denoting by $\hat{V}(\bar{D})=\sum_{t=1}^{T} g\left(\bar{D}_{t}\right)$ and $\hat{V}\left(\bar{D}^{*}\right)=\sum_{t=1}^{T} g\left(\bar{D}_{t}^{*}\right)=$ $V\left(\bar{D}^{*}\right)$ the corresponding functional values, we claim:

$$
\hat{V}\left(\bar{D}^{*}\right)=\sum_{t=1}^{T} g\left(\bar{D}_{t}^{*}\right) \leq \sum_{t=1}^{T} g\left(\bar{D}_{t}\right)=\hat{V}(\bar{D}) .
$$

Considering expression (21) for $V$, it can be seen that (54) implies (24). In fact, $V$ is invariant with respect to the chosen time reordering, we have established that (53a) is fulfilled for any $\bar{D}=\bar{D}(u)$ with $u \in \mathscr{U}$ and (53b) is always satisfied by $\bar{D}=\bar{D}(u)$ and $\bar{D}^{*}=\bar{D}\left(u^{*}\right)$ when $u, u^{*} \in \mathscr{U}$, as the total demand over time is the same. To prove (54), we introduce $\bar{D}^{(0)}=\bar{D}$ and the difference $C^{(0)}(t)=\bar{D}_{t}^{(0)}-\bar{D}_{t}^{*}$. In the nontrivial case of different $\bar{D}^{*}$ and $\bar{D}$, there always exists $x^{(0)}$ defined as the minimum $t$ for which it holds:

$$
\begin{array}{rlrl}
C^{(0)}(t) & >0 & \\
C^{(0)}(t+1) & \leq 0 \quad \text { if } t<T .
\end{array}
$$

To see this, consider that (53) is equivalent to:

$$
\begin{aligned}
\sum_{l=1}^{t} C^{(0)}(l) \leq 0 \quad t & =1, \ldots, T-1 \\
\sum_{l=1}^{T} C^{(0)}(l) & =0 .
\end{aligned}
$$

Therefore, the last non-zero term in $C^{(0)}$ is always positive. The following non-empty sets are now defined:

$$
\begin{aligned}
& \mathscr{T}_{-}^{(0)}:=\left\{t: C^{(0)}(t)<0, t \leq x^{(0)}\right\} \\
& \mathscr{T}_{+}^{(0)}:=\left\{t: C^{(0)}(t)>0, t \leq x^{(0)}\right\} .
\end{aligned}
$$

From (56) and the definition of $x^{(0)}$, it always holds:

$$
t_{1}<t_{2} \quad \forall t_{1} \in \mathscr{T}_{-}^{(0)} \quad \forall t_{2} \in \mathscr{T}_{+}^{(0)} .
$$

We introduce now a different profile $\bar{D}^{(1)}$ which guarantees a reduction of the cost functional $\hat{V}$ with respect to $\bar{D}^{(0)}$. To this end, the following quantities are considered:

$$
\begin{gathered}
x_{-}^{(0)}=\underset{t \in \mathscr{T}_{-}^{(0)}}{\arg \min } C^{(0)}(t) \quad x_{+}^{(0)}=\underset{t \in \mathscr{T}_{+}^{(0)}}{\arg \max } C^{(0)}(t) \\
\delta^{(0)}=\min \left\{\left|C^{(0)}\left(x_{-}^{(0)}\right)\right|,\left|C^{(0)}\left(x_{+}^{(0)}\right)\right|\right\}
\end{gathered}
$$

The new profile $\bar{D}^{(1)}$ can then be defined as:

$$
\bar{D}_{t}^{(1)}=\bar{D}_{t}^{(0)}+\boldsymbol{\delta}^{(0)} \cdot \mathbb{1}_{\left\{x_{-}^{(0)}\right\}}(t)-\delta^{(0)} \cdot \mathbb{1}_{\left\{x_{+}^{(0)}\right\}}(t) .
$$

The inequality $\hat{V}\left(\bar{D}^{(1)}\right) \leq \hat{V}\left(\bar{D}^{(0)}\right)$ can be demonstrated as in the proof of Proposition 2, showing that the equivalent of (29) holds for $\bar{D}^{(0)}$ and $\bar{D}^{(1)}$. The procedure detailed above can be repeated for increasing values of $i$, ensuring that at each step $\hat{V}\left(\bar{D}^{(i+1)}\right) \leq \hat{V}\left(\bar{D}^{(i)}\right)$. If $\delta^{(i)}$ is chosen through (59) evaluated at superscript $(i)$, at each iteration the cardinality of the support of $C^{(i)}$ is reduced by one. Thus, there exists $\alpha \leq$ $T$ such that $\bar{D}^{(\alpha)}=\bar{D}^{*}$. Condition (54), implying the theorem statement, is verified from the following inequalities:

$$
\hat{V}\left(\bar{D}^{*}\right)=\hat{V}\left(\bar{D}^{(\alpha)}\right) \leq \hat{V}\left(\bar{D}^{(\alpha-1)}\right) \leq \cdots \leq \hat{V}\left(\bar{D}^{(0)}\right)=\hat{V}(\bar{D}) .
$$

\section{REFERENCES}

[1] G. Strbac, "Demand side management: Benefits and challenges," Energy Policy, vol. 36, no. 12, pp. 4419-4426, 2008.

[2] M. Albadi and E. El-Saadany, "A summary of demand response in electricity markets," Electric Power Systems Research, vol. 78, no. 11, pp. 1989-1996, 2008.

[3] C. L. Su and D. Kirschen, "Quantifying the effect of demand response on electricity markets," IEEE Transactions on Power System, vol. 24, no. 3, pp. 1199-1207, 2009.

[4] D. Papadaskalopoulos and G. Strbac, "Decentralized participation of flexible demand in electricity markets - part I: Market mechanism," IEEE Trans. on Power Systems, vol. 28, no. 4, pp. 3658-3666, 2013.

[5] S. Ruthe, C. Rehtanz, and S. Lehnhoff, "On the problem of controlling shiftable prosumer devices with price signals," in Power Systems Computation Conference (PSCC), 2014, 2014, pp. 1-7.

[6] J. Rivera, C. Goebel, and H. Jacobsen, "Distributed convex optimization for electric vehicle aggregators," IEEE Transactions on Smart Grid, vol. PP, no. 99, 2016.

[7] L. Gan, U. Topcu, and S. H. Low, "Optimal decentralized protocol for electric vehicle charging," IEEE Transactions on Power Systems, vol. 28, no. 2, pp. 940-951, 2013.

[8] Z. Ma, D. Callaway, and I. Hiskens, "Decentralized charging control of large populations of plug-in electric vehicles," IEEE Transactions on Control Systems Technology, vol. 21, no. 1, pp. 67-78, 2013.

[9] Z. Ma, S. Zou, L. Ran, X. Shi, and I. Hiskens, "Efficient decentralized coordination of large-scale plug-in electric vehicle charging," Automatica, vol. 69, pp. 35-47, 2016.

[10] H. Chen, Y. Li, R. Louie, and B. Vucetic, "Autonomous demand side management based on energy consumption scheduling and instantaneous load billing: An aggregative game approach," IEEE Transactions on Smart Grid, vol. 5, no. 4, pp. 1744-1754, 2014.

[11] D. Paccagnan, M. Kamgarpour, and J. Lygeros, "On aggregative and mean field games with applications to electricity markets," in 2016 European Control Conference (ECC), 2016, pp. 196-201.

[12] L. Deori, K. Margellos, and M. Prandini, "On the connection between nash equilibria and social optima in electric vehicle charging control games," in 2017 IFAC World Congress, 2017, pp. 14 885-14 890.

[13] A. D. Paola, D. Angeli, and G. Strbac, "Price-based schemes for distributed coordination of flexible demand in the electricity market," IEEE Transactions on Smart Grid, vol. PP, no. 99, pp. 1-1, 2017.

[14] National Grid. (2016, Mar.) Historical demand data. [Online]. Available: http://www2.nationalgrid.com/UK/Industry-information /Electricity-transmission-operational-data/Data-Explorer/

[15] C. D. Aliprantis and K. C. Border, Infinite Dimensional Analysis. Springer, 2007.

[16] R. Goebel, R. G. Sanfelice, and A. R. Teel, Hybrid Dynamical Systems. Princeton University Press, 2012.

[17] A. L. Dontchev and R. T. Rockafellar, Implicit Functions and Solution Mappings. Springer, 2009. 